\title{
Determining the Singularities for the Observation of Three Image Lines
}

\author{
Sébastien Briot ${ }^{1}$, Philippe Martinet ${ }^{2}$ and François Chaumette ${ }^{3}$
}

\begin{abstract}
The determination of the singularity cases in the observation of image features is a complicated problem which is still open, apart from image points. For the first time, we provide the singularity cases in the observation of three image lines. We show that a concept named the "hidden robot", which was formerly used for understanding the singularities of a visionbased controller dedicated to parallel robots and, more recently which proved to be efficient for finding the singularity cases in the observation of image points, can be used for interpreting the singularities in the observation of three image lines. The hidden robot concept considerably simplifies the analysis by using geometric interpretations of the mapping degeneracy and tools provided by the mechanical engineering community. We prove that in the most complicated case where three general lines in space are observed, singularities appear when the origin of the observed object frame is either on a quadric or a cubic surface whose location in space depends on the configuration of the observed lines. In simpler cases where at least two lines belong to the same plane, these two surfaces can degenerate into simpler geometrical loci (e.g. planes, cylinders, lines).
\end{abstract}

Index Terms-Visual Servoing, Kinematics

\section{INTRODUCTION}

$\mathbf{F}$ INDING the singularity cases in the observation of image features is crucial in visual servoing and pose estimation [1]. However, this problem is still unsolved for most of the cases due to its considerable complexity. The singularities were found in the case of three image points after rather complicated mathematical computations [2], but they are still unknown for other image features.

In order to avoid the controllability issues near singularities, authors usually propose to observe additional features. However this leads to a non-minimal representation of the interaction between the system and the camera, resulting in the potential appearance of local minima [3] which are also difficult to determine. Additionally, even adding visual features may not ensure the absence of singularity cases [4]. Therefore, finding the singularities is crucial but it is usually prevented by the complexity of the mathematical problem to solve.

Manuscript received: July, 17, 2016; Revised October, 20, 2016; Accepted November, 17, 2016.

This paper was recommended for publication by Editor Jana Kosecka upon evaluation of the Associate Editor and Reviewers' comments.

This work was supported by the project RobEcolo funded by the French Région Pays de la Loire (Convention No. 2015-10773).

${ }^{1} \mathrm{~S}$. Briot is with CNRS at the Institut de Recherche en Communications et Cybernétique de Nantes (IRCCyN), UMR CNRS 6597, Nantes, France. Sebastien.Brioteirccyn.ec-nantes.fr

${ }^{2} \mathrm{P}$. Martinet is with the École Centrale de Nantes at IRCCyN, Nantes, France. Philippe. Martineteirccyn.ec-nantes.fr

${ }^{3} \mathrm{~F}$. Chaumette is with Inria at IRISA, Rennes, France. Francois.Chaumettedinria.fr
Recently, a concept named the "hidden robot" was introduced in [5], [6]. This concept was first used to determine the singularity cases of a vision-based controller dedicated to parallel robots [7] but it was recently proven that it can be extended to more general cases, such as the observation of $n$ image points [8].

The basic idea shown in [6], [8] is that the singularity cases of the mappings involved in the visual observation of geometric primitives for estimating the pose of an object or for visual servoing are equivalent to mappings representing the geometric and kinematic properties of given hidden parallel robots. By geometric property, we mean that the solutions of the Forward Geometric Model (FGM) of the parallel robots under consideration are also the solutions of the 3-D localization problem associated with the considered observations. By kinematic property, we mean that the singularities of the inverse kinematic Jacobian matrix of the robot are the same as the singularities of the interaction matrix that defines the link between the time variation of the visual features and the camera velocity. The hidden robot is indeed a tangible visualization of the mapping between the observation space and the Cartesian space. A methodology is proposed in [6] in order to define the hidden robot models associated with visual servoing dedicated to parallel robots while [8] presents an approach for finding the virtual robot models associated with more general cases based on the observation of geometric visual primitives.

By finding this correlation, it is then possible to study the singularities of the interaction matrix, by using advanced tools coming from the mechanical engineering community (e.g. the Grassmann-Cayley algebra [9] and/or the Grassmann geometry [10]). The interest in using these tools is that they are (most of the time) able to provide simple geometric interpretations of the singularity cases. However, it should be noted that these tools still require an experienced user.

In the present paper, we show that a virtual parallel robot is hidden within the mapping used in the observation of image lines [11] and, thanks to this correlation, we analyze the singularity cases when three lines are observed. To the best of our knowledge, this is the first time that this problem is solved, except for obvious cases such as when the three 3-D lines are parallel (leading to an unconstrained translation along the direction of the lines).

As a result, the paper is organized as follows. Next section shows that the geometric / kinematic mapping involved in the observation of image lines is the same as the mapping 


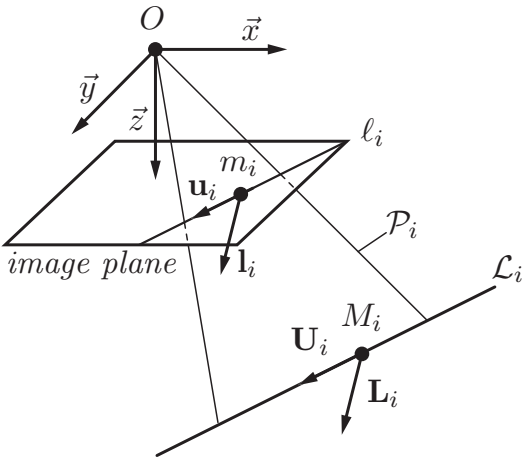

Fig. 1. Observation of a line

required to control a particular 3-UPRC parallel $\operatorname{robot}^{1}$. Then, in Section III, the singularities of the mapping are analyzed and validated through simulations. Finally, conclusions are drawn in Section IV.

\section{THE ROBOT MODEL HIDDEN IN THE OBSERVATION OF LINES}

Before presenting the architecture of robot hidden in the observation of three lines, we make some brief recalls on the computation of the related interaction matrix.

\section{A. Interaction matrix of three image lines}

In the following section of the paper, we use the standard pin hole model with focal length equal to 1 for the representation of the camera model. However, any other model based on projective geometry could be used.

A 3-D line $\mathcal{L}_{i}$ of Plücker coordinates $\left[\mathbf{U}_{i}^{T} \mathbf{L}_{i}^{T}\right]^{T}$ in the camera frame $\left(\mathbf{U}_{i}\right.$ being a unit vector characterizing its direction while $\mathbf{L}_{i}=\mathbf{U}_{i} \times \mathbf{X}, \mathbf{X}$ being the coordinates of any point $M_{i}$ belonging to $\mathcal{L}_{i}$ ) is projected in the image plane on a 2-D line $\ell_{i}$ of Plücker coordinates $\left[\mathbf{u}_{i}^{T} \mathbf{l}_{i}^{T}\right]^{T}\left(\mathbf{u}_{i}\right.$ being a unit vector characterizing its direction while $\mathbf{l}_{i}=\mathbf{u}_{i} \times \mathbf{x}, \mathbf{x}$ being the coordinates of any point $m_{i}$ belonging to $\ell_{i}$ ) (Fig. 1). From [12], we know that

$$
\mathbf{u}_{i}=\left[\begin{array}{l}
u_{x i} \\
u_{y i} \\
u_{z i}
\end{array}\right]=\left[\begin{array}{c}
L_{y i} / \Delta \\
-L_{x i} / \Delta \\
0
\end{array}\right], \mathbf{l}_{i}=\left[\begin{array}{l}
l_{x i} \\
l_{y i} \\
l_{z i}
\end{array}\right]=\left[\begin{array}{c}
L_{x i} / \Delta \\
L_{y i} / \Delta \\
L_{z i} / \Delta
\end{array}\right]
$$

where $\mathbf{L}_{i}=\left[L_{x i} L_{y i} L_{z i}\right]^{T}$ and $\Delta=\sqrt{L_{x i}^{2}+L_{y i}^{2}}$.

By differentiating these equations, the classical equations linking the velocities $\dot{\mathrm{i}}_{i}$ (and as a result the velocities $\dot{\mathbf{u}}_{i}$ ) to the twist $\boldsymbol{\tau}_{c}^{T}=\left[\boldsymbol{v}_{c}^{T} \boldsymbol{\omega}_{c}^{T}\right]$ of the camera in its relative motion with respect to the observed object frame ( $\boldsymbol{v}_{c}$ being the translational velocity and $\boldsymbol{\omega}_{c}$ the rotational velocity expressed in the camera frame) are

$$
\mathrm{i}_{i}=\mathbf{M}_{i} \boldsymbol{\tau}_{c}
$$

\footnotetext{
${ }^{1}$ In the following of the paper, $P, U, R, C$ will stand for passive prismatic, universal, revolute and cylindrical joints, respectively. If the letter is underlined, the joint is considered active.
}

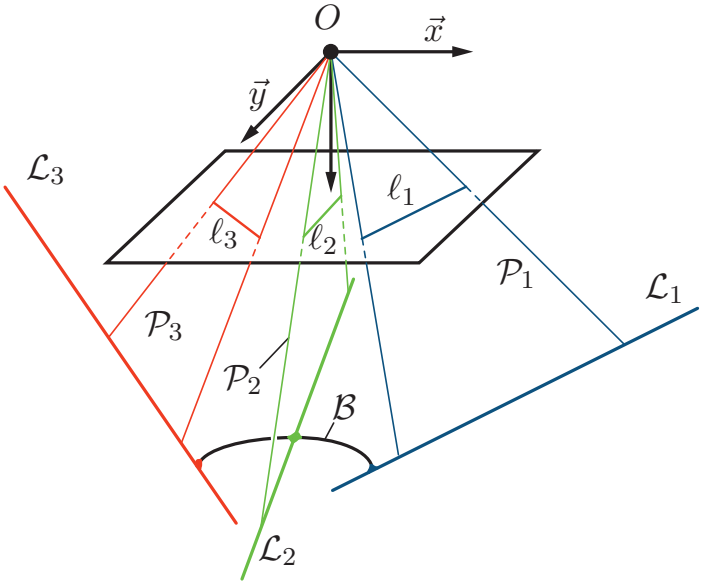

Fig. 2. Observation of three lines lying on a body $\mathcal{B}$

where $\mathbf{M}_{i}=\left[\mathbf{M}_{i 1}^{T} \mathbf{M}_{i 2}^{T} \mathbf{M}_{i 3}^{T}\right]^{T}$ with

$$
\begin{aligned}
& \mathbf{M}_{i 1}=\left[\begin{array}{c}
l_{x i} l_{y i} U_{z i} / \Delta \\
l_{y i}^{2} U_{z i} / \Delta \\
-l_{y i}\left(l_{x i} U_{x i}+l_{y i} U_{y i}\right) / \Delta \\
l_{x i} l_{y i} l_{z i} \\
l_{y i}^{2} l_{z i} \\
-l_{y i}
\end{array}\right]^{T} \\
& \mathbf{M}_{i 2}=\left[\begin{array}{c}
-l_{x i}^{2} U_{z i} / \Delta \\
-l_{x i} l_{y i} U_{z i} / \Delta \\
l_{x i}\left(l_{x i} U_{x i}+l_{y i} U_{y i}\right) / \Delta \\
-l_{x i}^{2} l_{z i} \\
-l_{x i} l_{y i} l_{z i} \\
l_{x i}
\end{array}\right]^{T} \\
& \mathbf{M}_{i 3}=\left[\begin{array}{c}
\left(U_{y i}+l_{y i} l_{z i} U_{z i}\right) / \Delta \\
-\left(U_{x i}+l_{x i} l_{z i} U_{z i}\right) / \Delta \\
l_{z i}\left(l_{x i} U_{y i}-l_{y i} U_{x i}\right) / \Delta \\
l_{y i}\left(l_{z i}^{2}+1\right) \\
-l_{x i}\left(l_{z i}^{2}+1\right) \\
0
\end{array}\right]^{T}
\end{aligned}
$$

In order to fully servo the relative motion between an object and a camera, at least three lines fixed on the object must be observed [11] (Fig. 2). Thus, considering the observation of three lines $\mathcal{L}_{1}, \mathcal{L}_{2}$ and $\mathcal{L}_{3}$, the interaction matrix linking the velocities $\dot{i}_{i}$ of the lines $\ell_{i}(i=1,2,3)$ grouped in the vector $\dot{\mathbf{s}}=\left[\begin{array}{lll}\mathbf{l}_{1}^{T} & \mathbf{l}_{2}^{T} & \mathbf{i}_{3}^{T}\end{array}\right]^{T}$ to the camera twist $\boldsymbol{\tau}_{c}$ by the relation

$$
\dot{\mathbf{s}}=\mathbf{M} \tau_{c}
$$

is thus given by

$$
\mathbf{M}=\left[\begin{array}{lll}
\mathbf{M}_{1}^{T} & \mathbf{M}_{2}^{T} & \mathbf{M}_{3}^{T}
\end{array}\right]^{T}
$$

Singularities appear when the matrix $\mathbf{M}$ is rank deficient. Looking at the analytical form of $\mathbf{M}$, it is clear that determining the singularities of $\mathbf{M}$ through the determinant of $\mathbf{M}^{T} \mathbf{M}$ seems to be out of reach.

Note that we have used above the Plücker representation of lines, but following results are not dependent of the choice of the representation (Cartesian, cylindrical) [12]. 


\section{B. Hidden robot model}

In this section, we show that a virtual parallel robot is hidden within the mapping used in the observation of image lines by considering what follows. First, from the single measure of the location of a line $\ell_{i}$ in the image plane, it is impossible to know the location of its corresponding 3-D line $\mathcal{L}_{i}$. The only information that we can extract from this measure is that the 3D line $\mathcal{L}_{i}$ lies on the interpretation plane $\mathcal{P}_{i}$ passing through $\ell_{i}$ and the optical center $O$. As a result, the measure of $\ell_{i}$ gives the location of the plane $\mathcal{P}_{i}$. Furthermore, from the same single measure, the orientation of the observed body $\mathcal{B}$ cannot be deduced (we need the three measures of the positions of lines $\ell_{1}, \ell_{2}$ and $\ell_{3}$, see Fig. 2).

From a mechanical engineer point of view, these geometric properties can be obtained by the kinematic architecture depicted in Fig. 3. This architecture is made of an actuated cardan (or universal $(\underline{U})$ ) joint rotating around point $O$ and fixed on the camera frame at that point. The $\underline{U}$ joint is followed by a passive prismatic $(P)$ joint whose direction $z_{i}$ is reciprocal to the axes $\boldsymbol{x}_{i}$ and $\boldsymbol{y}_{i}$ of the cardan joint. Then, the passive $P$ joint is attached at its other extremity to a passive revolute $(R)$ joint whose axis is directed along $\boldsymbol{y}_{i}$. Finally, the last link (moving platform, equivalent to the observed body $\mathcal{B}$ ) is connected to the $R$ joint by a passive cylindrical $(C)$ joint whose axis $\boldsymbol{a}_{i}$ is orthogonal to the axis of the $R$ joint. Thus, we have a leg with a $\underline{U} P R C$ architecture linking the camera frame to the observed object frame. To this leg, we associate a vector $\mathbf{q}_{i}=\left[\alpha_{i} \beta_{i}\right]^{T}$, where $\alpha_{i}$ and $\beta_{i}$ represent the rotation angles of each revolute joints composing the $\underline{U}$ joint (Fig. 3). With such a leg, the observed body $\mathcal{B}$ is constrained to translate along $\mathcal{L}_{i}$ and to rotate around it. As a result, the vectors $\mathbf{U}_{i}$ and $\mathbf{L}_{i}$ associated with the Plücker coordinates of the line $\mathcal{L}_{i}$ are collinear with the axes $\boldsymbol{a}_{i}$ and $\boldsymbol{y}_{i}$, respectively (Fig. 3).

It should be mentioned that the rotational component of the $C$ joint in this leg is necessary because we know that line $\mathcal{L}_{i}$ is attached to a rigid element (the observed body $\mathcal{B}$ ) whose orientation cannot be defined by considering a single measure $\ell_{i}$. Without taking into account this information, the displacement of the line $\mathcal{L}_{i}$ can be obtained by a leg with a $\underline{U} P R$ architecture, i.e. a kinematic chain with two passive degrees of freedom (instead of four for the $\underline{U P R C} \mathrm{leg}$ ).

Considering now that:

- the three observed 3-D lines $\mathcal{L}_{1}, \mathcal{L}_{2}$ and $\mathcal{L}_{3}$ have motions whose geometric properties can be parameterized, for each of them, by a $\underline{U P R C}$ mechanical architecture depicted above,

- $\mathcal{L}_{1}, \mathcal{L}_{2}$ and $\mathcal{L}_{3}$ are fixed on the same body $\mathcal{B}$,

- there is a global diffeomorphism between the configuration of the line $\ell_{i}$ and the $\underline{U}$ joint rotations $\mathbf{q}_{i}$; as a result, $\mathrm{l}_{i}$ is a singularity-free observation of the $\underline{U}$ joint motions $\mathbf{q}_{i}$,

then the relative motion between the body $\mathcal{B}$ and the camera frame has the same geometric and kinematic properties as the motion of a $3-\underline{U} P R C$ parallel robot (Fig. 4), i.e.

- the solutions of the FGM of the $3-\underline{U} P R C$ parallel robot are also the solutions of the 3-D localization problem

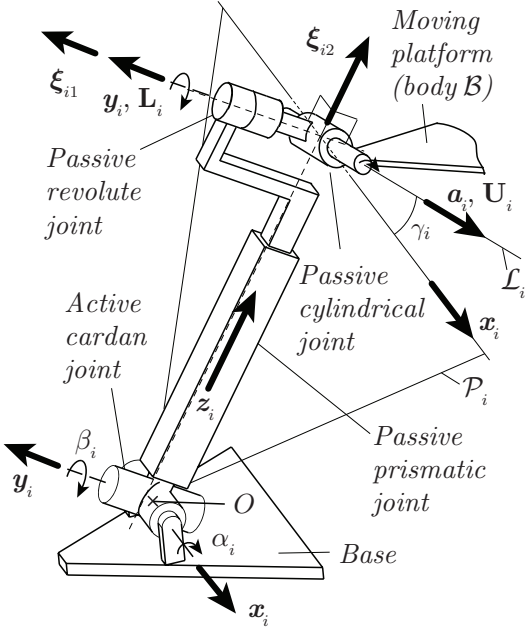

Fig. 3. A $\underline{U P R C}$ kinematic chain

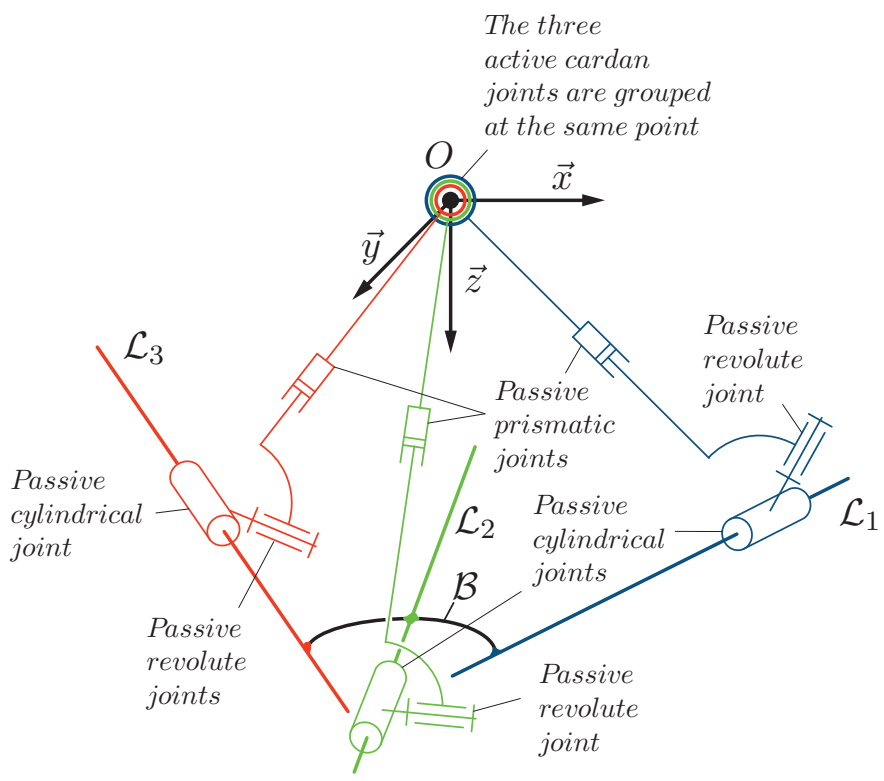

Fig. 4. The hidden robot model corresponding to the observation of three lines lying on a body $\mathcal{B}$ : a $3-\underline{U} P R C$ parallel robot with all active cardan joints merged at the point $O$ (for reason of clarity of drawings, the axes of the cardan joints are not represented)

when three 3-D lines are observed by a perspective camera.

- the singularities of the inverse kinematic Jacobian matrix of the robot are the same as the singularities of the interaction matrix (7).

To prove the last item, let us consider what follows. The inverse kinematic Jacobian matrix $\mathbf{J}_{i n v}$ of the robot links the actuators velocities $\dot{\mathbf{q}}=\left[\begin{array}{ll}\dot{\mathbf{q}}_{1}^{T} & \dot{\mathbf{q}}_{2}^{T} \\ \dot{\mathbf{q}}_{3}^{T}\end{array}\right]^{T}$ to the platform twist $\tau_{p}$ through the relation

$$
\dot{\mathbf{q}}=\mathbf{J}_{i n v} \boldsymbol{\tau}_{p}
$$

$\mathbf{J}_{i n v}$ is of dimension $(6 \times 6)$. Moreover, as mentioned above, there is a global diffeomorphism between the measure of the image line configuration $\ell_{i}$ and the $\underline{U}$ joint rotations $\mathbf{q}_{i}$. As a 
result,

$$
\dot{\mathbf{q}}=\mathbf{G} \dot{\mathbf{s}}
$$

where $\mathbf{G}$ is a $(6 \times 9)$ block-diagonal matrix which cannot be rank-deficient. Finally, by combining (6) and (9) with the screw transformation relation linking the camera twist $\boldsymbol{\tau}_{c}$ (expressed with respect to the object frame) to the observed object twist $\boldsymbol{\tau}_{p}$ (expressed with respect to the camera frame) by [3]

$$
\boldsymbol{\tau}_{c}=\mathbf{H} \boldsymbol{\tau}_{p}, \text { where } \mathbf{H}=\left[\begin{array}{cc}
-\mathbf{I}_{3} & {\left[{ }^{c} \mathbf{t}_{p}\right]_{\times}} \\
\mathbf{0}_{3 \times 3} & -\mathbf{I}_{3}
\end{array}\right]
$$

(in which $\mathbf{I}_{3}$ is the $(3 \times 3)$ identity matrix, $\mathbf{0}_{3 \times 3}$ is a $(3 \times 3)$ zero matrix, and $\left[{ }^{c} \mathbf{t}_{p}\right]_{\times}$is the cross-product matrix associated to the 3-D vector ${ }^{c} \mathbf{t}_{p}$ characterizing the translation between the camera and the observed object frames), we found:

$$
\mathbf{J}_{\text {inv }}=\mathbf{G M H}
$$

Then, as matrices $\mathbf{G}$ and $\mathbf{H}$ are never rank-deficient, we obligatorily have a loss of rank of $\mathbf{M}$ if and only if $\mathbf{J}_{i n v}$ is rank-deficient. Therefore, the conditions of singularity of the hidden robot inverse kinematic Jacobian matrix $\mathbf{J}_{i n v}$ are the same as the singularity conditions of the interaction matrix M.

\section{SINGULARITY ANALYSIS}

\section{A. General conditions of singularity}

Singularities on the inverse Jacobian matrix $\mathbf{J}_{i n v}$ of a parallel robot (also called Type 2 or parallel singularities [13]) appear when at least two solutions of the FGM are identical [10]. As mentioned in Section II, these singularities are analogous to the singularities of the interaction matrix.

In Type 2 singularities, parallel robots gain one (or more) uncontrollable motion. Kinematically speaking, there exists a non-null vector $\mathbf{t}_{s}$ defined such that $\mathbf{J}_{i n v} \mathbf{t}_{s}=\mathbf{0}$ while $\dot{\mathbf{q}}=\mathbf{0}$, i.e. the actuators are fixed (which means that $\mathbf{t}_{s}$ is in the null space of $\mathbf{J}_{i n v}$ ). As known in mechanics, if a rigid body got an uncontrollable motion, this means that it is not fully constrained by the system of wrenches applied on it, i.e. the static equilibrium is not ensured. As this uncontrollable motion appears only in a singularity, this means that locally the system of actuation wrenches, i.e. wrenches transmitted from the actuators to the platform by the legs, is degenerated [10].

For a given leg $i$, any actuation wrench denoted by $\boldsymbol{\xi}_{i j}$ is reciprocal to the unit twists denoted $\boldsymbol{\zeta}_{i k}$ characterizing the displacements of the passive joints [14], i.e. $\boldsymbol{\xi}_{i j}^{T} \boldsymbol{\zeta}_{i k}=0$ for any $j$ and $k$. This means that the virtual power developed by the wrench $\boldsymbol{\xi}_{i j}$ along the direction of motion $\boldsymbol{\zeta}_{i k}$ is null; in other words, the actuator $j$ of the leg $i$ cannot transmit a wrench $\boldsymbol{\xi}_{i j}$ to the platform along the direction $\boldsymbol{\zeta}_{i k}$.

Let us consider a $\underline{U} P R C$ leg belonging to our $3-\underline{U} P R C$ hidden robot. In the frame $\mathcal{F}_{i}:\left(M_{i}, \boldsymbol{x}_{i}, \boldsymbol{y}_{i}, \boldsymbol{z}_{i}\right)$ ( $M_{i}$ being the point of intersection between the axis of the prismatic and revolute joints) attached to the leg, the unit twist defining the motion of the passive $P$ joint is expressed as (see Fig. 3):

$$
\boldsymbol{\zeta}_{i 1}=\left[\begin{array}{llllll}
0 & 0 & 1 & 0 & 0 & 0
\end{array}\right]^{T}
$$

while

$$
\boldsymbol{\zeta}_{i 2}=\left[\begin{array}{llllll}
0 & 0 & 0 & 0 & 1 & 0
\end{array}\right]^{T}
$$

is the unit twist defining the motion of the passive $R$ joint and

$$
\begin{aligned}
\boldsymbol{\zeta}_{i 3} & =\left[\begin{array}{lllllll}
\cos \gamma_{i} & 0 & -\sin \gamma_{i} & 0 & 0 & 0
\end{array}\right]^{T} \\
\boldsymbol{\zeta}_{i 4} & =\left[\begin{array}{llllll}
0 & 0 & 0 & \cos \gamma_{i} & 0 & -\sin \gamma_{i}
\end{array}\right]^{T}
\end{aligned}
$$

are the unit twists defining the motions of the passive $C$ joint, $\gamma_{i}$ being the angle between $\boldsymbol{x}_{i}$ and $\boldsymbol{a}_{i}$ (and thus $\mathbf{U}_{i}$ ), both axes being contained in $\mathcal{P}_{i}$ (Fig. 3).

In these twists, the three first components represent the direction of the translation velocity while the three last components represent the direction of the rotational velocity.

As a result, the unit actuation wrenches $\boldsymbol{\xi}_{i j}=\left[\mathbf{f}_{i j}^{T} \mathbf{m}_{i j}^{T}\right]^{T}$ expressed in the frame $\mathcal{F}_{i}$ are

$$
\begin{gathered}
\boldsymbol{\xi}_{i 1}: \mathbf{f}_{i 1}=\left[\begin{array}{lll}
0 & 1 & 0
\end{array}\right]^{T}, \mathbf{m}_{i 1}=\mathbf{0}_{3 \times 1} \\
\boldsymbol{\xi}_{i 2}: \mathbf{f}_{i 2}=\mathbf{0}_{3 \times 1}, \mathbf{m}_{i 2}=\left[\begin{array}{lll}
\sin \gamma_{i} 0 & \cos \gamma_{i}
\end{array}\right]^{T}
\end{gathered}
$$

in which $\mathbf{f}_{i j}$ represents the direction of the force exerted on the platform and $\mathbf{m}_{i j}$ the direction of the moment. As a result, $\boldsymbol{\xi}_{i 1}$ is a pure force directed along $\boldsymbol{y}_{i}$ and $\boldsymbol{\xi}_{i 2}$ is a pure moment reciprocal to $\boldsymbol{\xi}_{i 1}$ and $\mathbf{U}_{i}$ which is included in the plane $\mathcal{P}_{i}$ (Fig. 3).

Thus, for the three robot legs, the system of actuation wrenches is given by $\boldsymbol{\xi}=\left[\boldsymbol{\xi}_{11} \boldsymbol{\xi}_{21} \boldsymbol{\xi}_{31} \boldsymbol{\xi}_{12} \boldsymbol{\xi}_{22} \boldsymbol{\xi}_{32}\right]$. There exists some tools able to define the conditions of degeneracy of a wrench system among which are the Grassmann geometry [10] and the Grassmann-Cayley algebra [9], [15]-[17]. In what follows, we use the Grassmann-Cayley algebra in order to find the singularity conditions related to our problem. Indeed, as any wrench of the wrench system $\xi$ can be seen as the Plücker representation of a line ${ }^{2}$ [10], the GrassmannCayley algebra makes it possible to compute the determinant of the wrench system $\boldsymbol{\xi}$ as an expression involving twelve points selected on the six lines (corresponding to the six wrenches). This expression is a linear combination of 24 monomials [9], each monomial representing the volume of a tetrahedron whose extremities correspond to four of the considered twelve points. By a smart selection of the twelve points, due to the experience of the user, it is possible to vanish a large number of the monomials and thus to simplify the expression of the determinant of the wrench system.

Regarding our particular case, the Grassmann-Cayley algebra was used in [17] to prove that, if the system of wrenches is composed of a pair of three forces $\boldsymbol{\xi}_{11}, \boldsymbol{\xi}_{21}, \boldsymbol{\xi}_{31}$ and of three moments $\boldsymbol{\xi}_{12}, \boldsymbol{\xi}_{22}, \boldsymbol{\xi}_{32}$, conditions of singularities appear if and only if:

$$
f_{1}=\mathbf{f}_{11}^{T}\left(\mathbf{f}_{21} \times \mathbf{f}_{31}\right)=0 \text { or } f_{2}=\mathbf{m}_{12}^{T}\left(\mathbf{m}_{22} \times \mathbf{m}_{32}\right)=0
$$

which means that the vectors $\mathbf{f}_{11}, \mathbf{f}_{21}$ and $\mathbf{f}_{31}\left(\mathbf{m}_{11}, \mathbf{m}_{21}\right.$ and $\mathbf{m}_{31}$, resp.) lie in the same plane (or are collinear to the same vector $\mathbf{c}$ ). As a result, the mechanism is not able to resist to forces $\mathbf{f}$ (moments $\mathbf{m}$, resp.) orthogonal to this plane (or in any directions orthogonal to c) and thus translations (rotations,

\footnotetext{
${ }^{2} \mathrm{~A}$ pure force will be represented by a line in the 3-D space while a pure moment will be a line in the projective space at infinity.
} 
resp.) are gained along the direction of $\mathbf{f}$ (around the axis of m, resp.).

From these two conditions, it is possible to find the configurations of the end-effector (the observed body) in the camera frame leading to singularities. Examples of singular configurations, depending on the arrangement of the lines on the observed body, are detailed below.

\section{B. Singularity loci}

Thanks to the particular geometric properties of the system to analyze (invariance of robot leg configurations for any rotation around $O$ ), it is possible to simplify the singularity analysis by fixing the platform orientation. Thus, all expressions below will be given for the "zero" platform orientation. For another given platform orientation (parameterized by the rotation matrix $\mathcal{R}$ ), singularity loci will be found by parameterizing the variables $X, Y$ and $Z$ characterizing the position of the origin of the object frame $\mathcal{F}_{b}$ in the camera frame (see Figs. 5 and 6) when considering the "zero" platform orientation thanks to new variables $X^{\prime}, Y^{\prime}$ and $Z^{\prime}$ representing the position of the origin of the object frame for the considered "non-zero" platform orientation such that

$$
\left[\begin{array}{lll}
X & Y & Z
\end{array}\right]^{T}=\mathcal{R}\left[\begin{array}{lll}
X^{\prime} & Y^{\prime} & Z^{\prime}
\end{array}\right]^{T}
$$

1) Three coplanar lines with no common intersection point: In such a case, the three lines intersect in points $M_{1}, M_{2}$ and $M_{3}$ (Fig. 5(a)) (we disregard the case where at least two lines are parallel, which is considered in Section III-B5). We define the frame $\mathcal{F}_{b}:\left(Q, \boldsymbol{x}_{b}, \boldsymbol{y}_{b}, \boldsymbol{z}_{b}\right)$ attached to the observed body $\mathcal{B}$ such that $Q$ is the center of the circumcircle of the triangle $\Delta M_{1} M_{2} M_{3}$ and $\boldsymbol{x}_{b}$ is collinear to $\overrightarrow{Q M_{1}}$. Moreover, for the analysis, we fix the orientation of the body so that $\mathcal{F}_{b}$ can be obtained from the camera frame by a translation of vector $\overrightarrow{O Q}$. As a result,

$$
\begin{aligned}
\overrightarrow{O Q} & =\left[\begin{array}{lll}
X Y Z & Z
\end{array}\right]^{T}, \overrightarrow{Q M_{1}}=\left[\begin{array}{ll}
\rho 0 & 0
\end{array}\right]^{T}, \\
\overrightarrow{Q M_{2}} & =\rho[\cos \phi \sin \phi 0]^{T}, \overrightarrow{Q M_{3}}=\rho[\cos \psi \sin \psi 0]^{T}
\end{aligned}
$$

where $\phi, \psi$ are two angles defined in Fig. 5(a) and $\rho$ is the radius of the circle.

Then, from section III-A, we know that

$$
\mathbf{f}_{i 1} \propto \mathbf{U}_{i} \times \overrightarrow{O M_{i}}, \mathbf{m}_{i 2} \propto \mathbf{U}_{i} \times \overrightarrow{\mathbf{f}_{i 1}}
$$

leading to, from (18), and after some tedious developments,

$$
\begin{aligned}
& f_{1}=0 \Leftrightarrow Z=0 \\
& f_{2}=0 \Leftrightarrow Z\left(X^{2}+Y^{2}-\rho^{2}\right)=0
\end{aligned}
$$

Condition $Z=0$ means that the plane containing the lines $\mathcal{L}_{1}$, $\mathcal{L}_{2}$ and $\mathcal{L}_{3}$ also contains the camera center $O$, which is not critical in practice. In such a case, all three lines project in a single line in the image plane and the pose of the object cannot be determined. Condition $X^{2}+Y^{2}=\rho^{2}$ means that the camera center $O$ lies on the cylinder whose axis is perpendicular to the plane containing all three points $M_{1}, M_{2}$ and $M_{3}$ and which includes the three points. These conditions are the same as the singularity cases where three image points are observed [2], which is indeed not a surprise due to the equivalence of both problems.

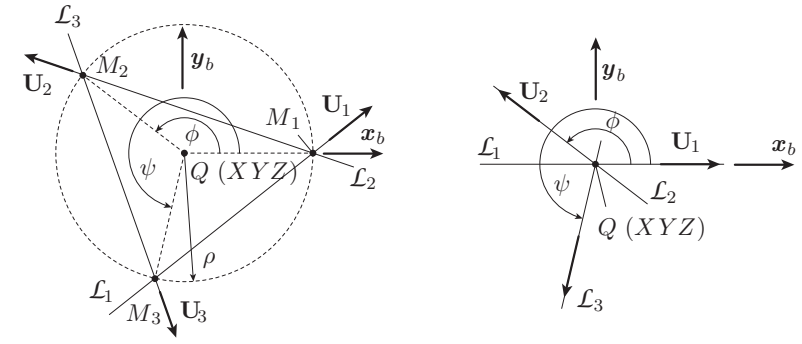

(a) with no common intersection (b) with a common intersection point point

Fig. 5. Observation of three coplanar lines

2) Three coplanar lines with a common intersection point: We define the frame $\mathcal{F}_{b}:\left(Q, \boldsymbol{x}_{b}, \boldsymbol{y}_{b}, \boldsymbol{z}_{b}\right)$ attached to the observed body $\mathcal{B}$ such that $Q$ is the intersection point of all three lines and $\boldsymbol{x}_{b}$ is collinear to $\mathrm{U}_{1}$ (Fig. 5(b)). Again, we fix the orientation of the body so that $\mathcal{F}_{b}$ can be obtained from the camera frame by a translation of vector $\overrightarrow{O Q}$. Thus,

$$
\begin{aligned}
& \overrightarrow{O Q}=\left[\begin{array}{lll}
X Y & Y Z
\end{array}\right]^{T}, \mathbf{U}_{1}=\left[\begin{array}{lll}
1 & 0 & 0
\end{array}\right]^{T}, \\
& \mathbf{U}_{2}=[\cos \phi \sin \phi 0]^{T}, \mathbf{U}_{3}=[\cos \psi \sin \psi 0]^{T}
\end{aligned}
$$

where $\phi, \psi$ are two angles defined in Fig. 5(b).

Then, from section III-A,

$$
\mathbf{f}_{i 1} \propto \mathbf{U}_{i} \times \overrightarrow{O Q}, \mathbf{m}_{i 2} \propto \mathbf{U}_{i} \times \overrightarrow{\mathbf{f}_{i 1}}
$$

leading to, from (18),

$$
\begin{aligned}
& f_{1}=0 \text { for any object configuration } \\
& f_{2}=0 \Leftrightarrow Z\left(X^{2}+Y^{2}\right)=0
\end{aligned}
$$

Thus, if three coplanar lines intersect in a single point, the rank of the interaction matrix is never full, i.e. at least one degree of freedom (a translation towards the intersection point) of the object cannot be controlled, as already known [11]. Moreover, additional degrees of freedom become uncontrollable if

- $Z=0$, which means as previously that the plane containing the lines $\mathcal{L}_{1}, \mathcal{L}_{2}$ and $\mathcal{L}_{3}$ also contains the camera center $O$.

- $X^{2}+Y^{2}=0$, which means that the camera center $O$ lies on the line which passes through $Q$ and which is perpendicular to all vectors $\mathbf{U}_{i}$, which is the same condition as when $f_{1}=0$.

3) Three lines in space with a common intersection point: Here, the three lines are spatial, not orthogonal and intersect in a common point. We define the frame $\mathcal{F}_{b}:\left(Q, \boldsymbol{x}_{b}, \boldsymbol{y}_{b}, \boldsymbol{z}_{b}\right)$ attached to the observed body $\mathcal{B}$ such that $\boldsymbol{x}_{b}$ is collinear to $\mathbf{U}_{1}, \boldsymbol{y}_{b}$ is in the plane containing $\mathbf{U}_{1}$ and $\mathbf{U}_{2}$, while $Q$ is the intersection point of the lines. As a result,

$$
\begin{aligned}
\overrightarrow{O Q} & =\left[\begin{array}{lll}
X Y Z & Y
\end{array}\right]^{T}, \mathbf{U}_{1}=\left[\begin{array}{lll}
1 & 0 & 0
\end{array}\right]^{T}, \\
\mathbf{U}_{2} & =\left[\begin{array}{lll}
a & b & 0
\end{array}\right]^{T}, \mathbf{U}_{3}=\left[\begin{array}{cc}
c & d e
\end{array}\right]^{T}
\end{aligned}
$$

where $a, b, c, d$ and $e$ are variables parameterizing the direction of the lines. 


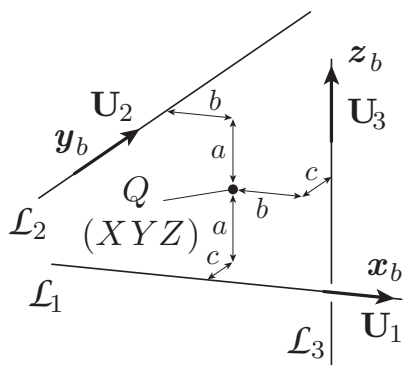

(a) no common intersection point

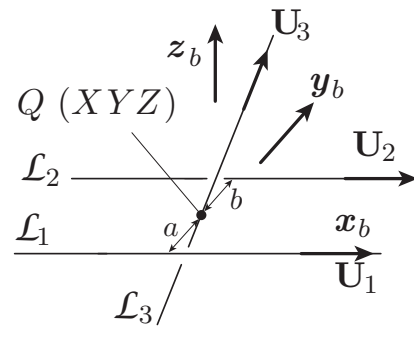

(b) two lines are parallel
Fig. 6. Observation of lines in space

$\mathbf{f}_{i 1}$ and $\mathbf{m}_{i 2}$ can be found thanks to expression (24), and from (18) we obtain,

$$
\begin{aligned}
f_{1} & =0 \text { for any object configuration } \\
f_{2} & =0 \Leftrightarrow b\left(a d e Y^{3}+\left(\left(-a d^{2}+b c d+a e^{2}\right) Z\right.\right. \\
& +(a c-b d) e X) Y^{2}-e\left(b c X^{2}+(a d-b c) Z^{2}\right. \\
& +2 b e X Z) Y+\left(\left(-a d^{2}+b c d-a e^{2}\right) X^{2} Z\right. \\
& \left.\left.+(b d+a c) e X Z^{2}\right)\right)=0
\end{aligned}
$$

Thus, if three spatial lines intersect in a single point, the rank of the interaction matrix is never full, i.e. at least one degree of freedom (a translation) of the object cannot be controlled, as already known [11]. Moreover, additional degrees of freedom become uncontrollable if the origin of the body frame belongs to a cubic surface parameterized by $f_{2}=0$.

Note also that in the case where the three lines are orthogonal, the equation $f_{2}=0$ degenerates into $X Y Z=0$.

4) Three orthogonal lines in space: Here, the three lines are orthogonal and do not intersect. We define the frame $\mathcal{F}_{b}$ : $\left(Q, \boldsymbol{x}_{b}, \boldsymbol{y}_{b}, \boldsymbol{z}_{b}\right)$ attached to the observed body $\mathcal{B}$ such that $\boldsymbol{x}_{b}$ is collinear to $\mathbf{U}_{1}, \boldsymbol{y}_{b}$ is collinear to $\mathbf{U}_{2}$ and $z_{b}$ is collinear to $\mathbf{U}_{3}$. Moreover, the distance between $\mathcal{L}_{1}$ and $\mathcal{L}_{2}$ is equal to $2 a$, between $\mathcal{L}_{2}$ and $\mathcal{L}_{3}$ is equal to $2 b$, and between $\mathcal{L}_{1}$ and $\mathcal{L}_{3}$ is equal to $2 c$, while $Q$ is the barycenter of the lines (Fig. 6(a)). As a result,

$$
\begin{aligned}
& \overrightarrow{O P_{1}}=[X(Y-c)(Z-a)]^{T}, \mathbf{U}_{1}=\left[\begin{array}{lll}
1 & 0 & 0
\end{array}\right]^{T} \\
& \overrightarrow{O P_{2}}=[(X-b) Y(Z+a)]^{T}, \mathbf{U}_{2}=\left[\begin{array}{lll}
0 & 1 & 0
\end{array}\right]^{T} \\
& \overrightarrow{O P_{3}}=[(X+b)(Y+c) Z]^{T}, \mathbf{U}_{3}=\left[\begin{array}{lll}
0 & 0 & 1
\end{array}\right]^{T}
\end{aligned}
$$

where $P_{i}$ is a point belonging to $\mathcal{L}_{i}$.

Then, we have

$$
\mathbf{f}_{i 1} \propto \mathbf{U}_{i} \times \overrightarrow{O P_{i}}, \mathbf{m}_{i 2} \propto \mathbf{U}_{i} \times \overrightarrow{\mathbf{f}_{i 1}}
$$

which leads to, from (18),

$$
\begin{aligned}
& f_{1}=0 \Leftrightarrow a X Y+b Y Z-c X Z-a b c=0 \\
& f_{2}=0 \Leftrightarrow a c X-a b Y+b c Z-X Y Z=0
\end{aligned}
$$

Expression $f_{1}$ represents a quadric surface while expression $f_{2}$ is a cubic surface (Fig. 7).

5) Three lines, two of them being parallel: Here, the lines $\ell_{1}$ and $\ell_{2}$ are parallel. We define the frame $\mathcal{F}_{b}:\left(Q, \boldsymbol{x}_{b}, \boldsymbol{y}_{b}, \boldsymbol{z}_{b}\right)$ attached to the observed body $\mathcal{B}$ such that $\boldsymbol{x}_{b}$ is collinear to $\mathbf{U}_{1}=\mathbf{U}_{2}, \boldsymbol{y}_{b}$ is lying in the plane $\mathcal{P}$ containing $\mathcal{L}_{1}$ and $\mathcal{L}_{2}$.

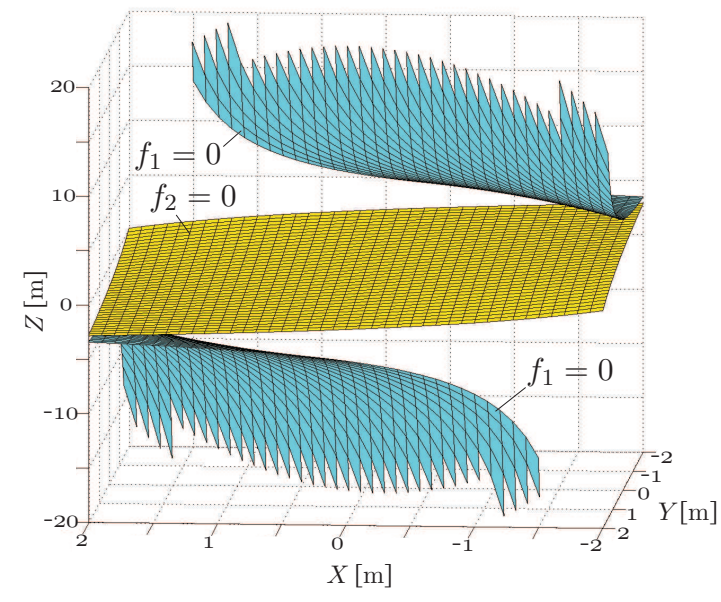

Fig. 7. Example of singularity loci when three perpendicular lines are observed (computed for $a=3.13, b=3.13, c=-3.13$ )

$Q$ is at the intersection of $\mathcal{P}$ and the line $\mathcal{L}_{3}$. From Fig. 6(b), we define

$$
\begin{aligned}
& \overrightarrow{O P_{1}}=[X(Y-a) Z]^{T}, \mathbf{U}_{1}=\left[\begin{array}{lll}
1 & 0 & 0
\end{array}\right]^{T} \\
& \overrightarrow{O P_{2}}=[X(Y+b) Z]^{T}, \mathbf{U}_{2}=\left[\begin{array}{lll}
1 & 0 & 0
\end{array}\right]^{T} \\
& \overrightarrow{O P_{3}}=\left[\begin{array}{lll}
X & Y & Z
\end{array}\right]^{T}, \mathbf{U}_{3}=\left[\begin{array}{ll}
c d e
\end{array}\right]^{T}
\end{aligned}
$$

where $c, d, e$ are variables parameterizing the direction of the line $\mathcal{L}_{3}$.

Then, $\mathbf{f}_{i 1}$ and $\mathbf{m}_{i 2}$ being found from (29), by using the singularity conditions (18), we obtain

$$
\begin{aligned}
& f_{1}=0 \Leftrightarrow Z(d Z-e Y)=0 \\
& f_{2}=0 \Leftrightarrow Z\left(X\left(d^{2}+e^{2}\right)-c Y d-c Z e\right)=0
\end{aligned}
$$

Here, singularities are equations of planes:

- $Z=0$, which occur when the plane $\mathcal{P}$ containing $\mathcal{L}_{1}$ and $\mathcal{L}_{2}$ also contains the optical center,

- $e Y-d Z=0$ is the plane containing $\mathbf{U}_{1}, \mathbf{U}_{3}$ and the optical center,

- $X\left(d^{2}+e^{2}\right)-c d Y-c e Z=0$ is the plane containing $\left(\mathbf{U}_{1} \times \mathbf{U}_{3}\right), \mathbf{U}_{3}$ and the optical center.

The two first cases are degenerated cases where two 3-D lines are contained in the same interpretation plane (i.e. the plane passing through the camera center and the image line), leading to the same image line for the two 3-D lines.

6) Three general lines in space: This is the most general case. We do not provide here the expressions which are more complex than in the previous cases but they are still exploitable and can be found in a technical report [18].

As in the case of the three perpendicular lines, condition $f_{1}=0$ provides the expression of a quadric surface while $f_{2}=0$ leads to a cubic surface.

\section{Illustrative examples}

1) Singularities when observing three general lines in space: In order to show the exactness of our results, we first perform simulations in the case where the three lines have a general configuration (Section III-B6). The three general 


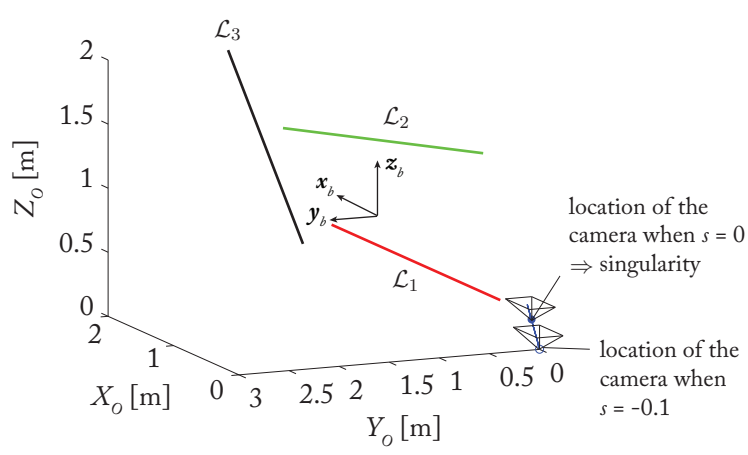

Fig. 8. Relative motion of the camera with respect to the observed lines in a general configuration.

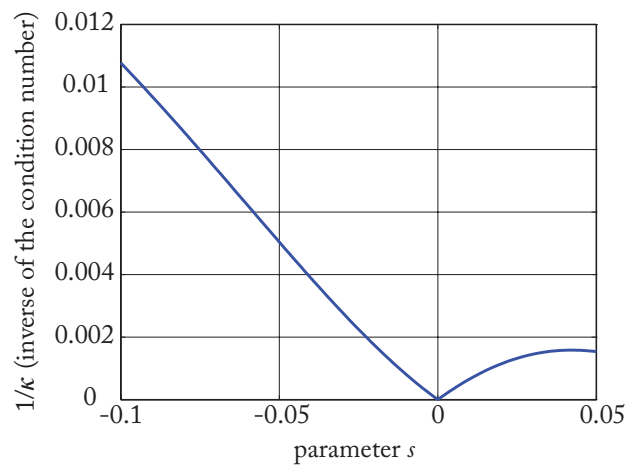

Fig. 9. Inverse of the condition number of the interaction matrix $\mathbf{M}$ obtained when observing three lines in the general case.

lines are parameterized as follows. We define the frame $\mathcal{F}_{b}:\left(Q, \boldsymbol{x}_{b}, \boldsymbol{y}_{b}, \boldsymbol{z}_{b}\right)$ attached to the observed body $\mathcal{B}$ such that $\boldsymbol{x}_{b}$ is collinear to $\mathbf{U}_{1}, \boldsymbol{y}_{b}$ is lying in the plane $\mathcal{P}$ containing $\mathcal{L}_{1}$ and $\mathcal{L}_{2}$. Then, the line $\mathcal{L}_{i}$ is parameterized by its direction $\mathbf{U}_{i}$ and a point $P_{i}$ lying on it by taking the following expressions:

$$
\begin{aligned}
& \overrightarrow{O P_{1}}=[(X-b)(Y-c)(Z-a)]^{T}, \mathbf{U}_{1}=\left[\begin{array}{lll}
1 & 0 & 0
\end{array}\right]^{T} \\
& \overrightarrow{O P_{2}}=[(X-b)(Y-c)(Z+a)]^{T}, \mathbf{U}_{2}=[d e 0]^{T} \\
& \overrightarrow{O P_{3}}=[(X+b)(Y+c) Z]^{T}, \mathbf{U}_{3}=[f g h]^{T}
\end{aligned}
$$

where $d, e, f, g$ and $h$ are variables parameterizing the direction of the lines $\mathcal{L}_{2}$ and $\mathcal{L}_{3}$, and $X, Y, Z$ are the coordinates of $Q$ in the camera frame. For simulation purpose, we set $a=b=c=1 \mathrm{~m}$ and $d=e=1, f=-1, g=h=1$. Then, a relative motion between the object frame and camera frame origins is imposed and is parameterized by the following functions characterizing the location of $Q$ in the camera frame:

$$
\begin{gathered}
X=1-0.5 s, Y=1-0.5 s, \\
Z=0.75-2.25 s
\end{gathered}
$$

with $s \in\left[\begin{array}{ll}-1 & 0.05\end{array}\right]$ a linearly increasing function. The relative motion of the camera with respect to the observed lines is shown in Fig. 8. In such simulation, when $s=0$, the point $O$ reaches the configuration $X_{O}=0.05 \mathrm{~m}, Y_{O}=0.05 \mathrm{~m}$ and $Z_{s}=0.225 \mathrm{~m}$ in the object frame (see Fig. 8) which is a point

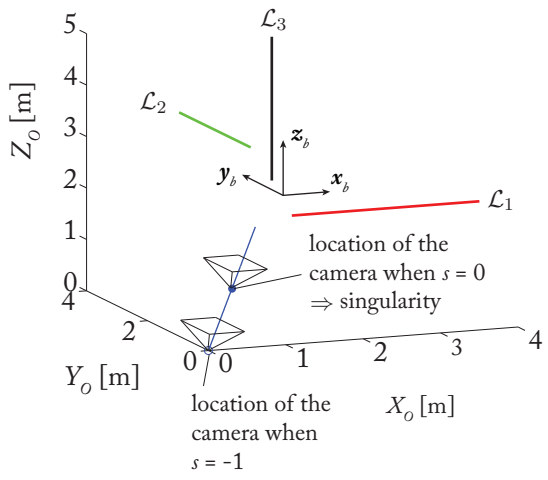

Fig. 10. Relative motion of the camera with respect to the three observed orthogonal lines.

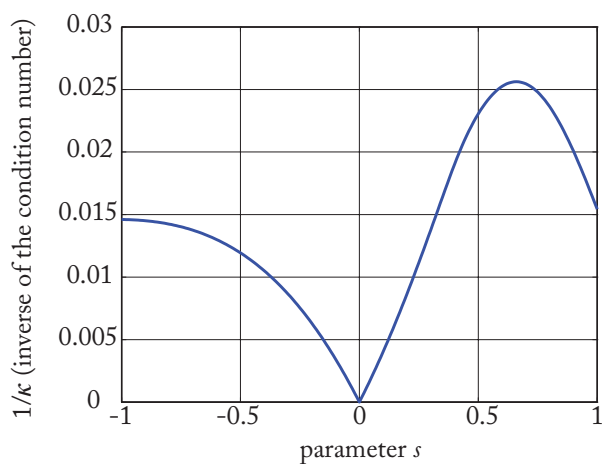

Fig. 11. Inverse of the condition number of the interaction matrix $\mathbf{M}$ obtained when observing three orthogonal lines.

lying on the quadric surface whose expression can be found in the technical report [18]. As a result, the rank-deficiency of the matrix $\mathbf{M}$ appears for $s=0$, which is shown on Fig. 9 in which the inverse of the condition number is null only for $s=0$.

2) Singularities when observing three orthogonal lines in space: A second simulation is performed in order to show now the singularities when observing three orthogonal lines in space (see Section III-B4). For simulation purpose, we take the following values of the parameters $a, b$ and $c$ in (28): $a=b=0.5 \mathrm{~m}, c=1.5 \mathrm{~m}$. Then, a relative motion between the object frame and camera frame origins is imposed and is parameterized by the following functions characterizing the location of $Q$ in the camera frame:

$$
\begin{gathered}
X=1-0.5 s, Y=1-0.5 s, \\
Z=2-s
\end{gathered}
$$

with $s \in\left[\begin{array}{ll}-1 & 1\end{array}\right]$ a linearly increasing function. The relative motion of the camera with respect to the observed lines is shown in Fig. 10. In such simulation, when $s=0$, the point $O$ reaches the configuration $X_{O}=0.5 \mathrm{~m}, Y_{O}=0.5 \mathrm{~m}$ and $Z_{O}=1 \mathrm{~m}$ in the object frame (see Fig. 10) which is a point lying on the cubic surface whose expression is given in (30). As a result, the rank-deficiency of the matrix $\mathbf{M}$ appears for 
$s=0$, which is shown on Fig. 11 in which the inverse of the condition number is null only for $s=0$.

\section{CONCLUSION}

In this paper, we determined the singularity cases for the observation of three image lines thanks to a tool named the "hidden robot concept". We showed that the hidden robot concept allows for considerable simplification of the analysis, leading to the computation of new singularity cases. Indeed, the hidden robot is a tangible visualization of the mapping between the observation space and the Cartesian space. As a result, the singular configurations of the hidden robot corresponded to the singularities of the interaction matrix.

Indeed, the concept of hidden robot allowed to change the way we defined the problem. By doing so, we were able to replace the degeneracy analysis of the velocity transmission between inputs (velocity of the observed features) and outputs (camera twist), by its dual but fully equivalent problem which was to analyze the degeneracy in the transmission of wrenches between the inputs of a virtual mechanical system (virtual actuators of the hidden robot whose displacement was linked to the motions of the observed features) and its outputs (wrenches exerted on the virtual platform, i.e. the observed object).

Then, by using geometric interpretations of the mapping degeneracy and tools provided by the mechanical engineering community such as the Grassmann-Cayley algebra, we were able to find rather simple geometric interpretation of the interaction matrix degeneracy. We proved that in the most complicated cases where three general lines in space are observed, singularities appear when the origin of the observed object frame is either on a quadric or a cubic surface. In simpler cases where at least two lines belong to the same plane, these two surfaces can degenerate into simpler geometrical loci (e.g. planes, cylinders, lines).

Future works concern the analysis of the singularity cases in the observation of $n$ lines, and research about the singularities when observing other primitives (spheres, circles, and eventually of combination of different primitives), or when using several cameras.

\section{REFERENCES}

[1] S. Hutchinson, G. Hager, and P. Corke, "A tutorial on visual servo control," IEEE Transactions on Robotics and Automation, vol. 12, pp. 651-670, 1996.

[2] H. Michel and P. Rives, "Singularities in the determination of the situation of a robot effector from the perspective view of 3 points," INRIA, Tech. Rep., 1993.

[3] F. Chaumette and S. Hutchinson, Visual Servoing and Visual Tracking, chapter 24 of Handbook of Robotics. Springer, 2008.

[4] V. Rosenzveig, S. Briot, P. Martinet, E. Özgür, and N. Bouton, "A method for simplifying the analysis of leg-based visual servoing of parallel robots," in Proc. 2014 IEEE Int. Conf. on Robotics and Automation (ICRA 2014), Hong Kong, China, May 2014.

[5] S. Briot and P. Martinet, "Minimal representation for the control of Gough-Stewart platforms via leg observation considering a hidden robot model," in 2013 IEEE Int. Conf. on Robotics and Automation (ICRA 2013), Karlsruhe, Germany, May, 6-10 2013.

[6] S. Briot, P. Martinet, and V. Rosenzveig, "The hidden robot: an efficient concept contributing to the analysis of the controllability of parallel robots in advanced visual servoing techniques," IEEE Transactions on Robotics, vol. 31, no. 6, pp. 1337-1352, 2015.
[7] N. Andreff, A. Marchadier, and P. Martinet, "Vision-based control of a Gough-Stewart parallel mechanism using legs observation," in 2005 IEEE Int. Conf. on Robotics and Automation (ICRA 2005), Barcelona, Spain, April 18-22 2005, pp. 2546-2551.

[8] S. Briot, F. Chaumette, and P. Martinet, "Revisiting the determination of the singularity cases in the visual servoing of image points through the concept of "hidden robot"," IEEE Transactions on Robotics, 2017, to appear.

[9] P. Ben-Horin and M. Shoham, "Singularity analysis of a class of parallel robots based on Grassmann-Cayley algebra," Mechanism and Machine Theory, vol. 41, no. 8, pp. 958-970, August 2006.

[10] J.-P. Merlet, Parallel Robots, 2nd ed. Springer, 2006, iSBN 978-14020-4133-4.

[11] N. Andreff, B. Espiau, and R. Horaud, "Visual servoing from lines," Int. Journal of Robotics Research, vol. 21, no. 8, pp. 679-700, 2002.

[12] F. Chaumette, "La relation vision-commande : théorie et application à des tâches robotiques," Ph.D. dissertation, Université de Rennes 1, 1990.

[13] C. Gosselin and J. Angeles, "Singularity analysis of closed-loop kinematic chains," IEEE Transactions on Robotics and Automation, vol. 6 , no. 3, pp. 281-290, 1990

[14] I. Bonev, "Geometric analysis of parallel mechanisms," Ph.D. dissertation, Université Laval, QC, Canada, nov 2002.

[15] P. Ben-Horin and M. Shoham, "Application of Grassmann-Cayley algebra to geometrical interpretation of parallel robot singularities," Int. Journal of Robotics Research, vol. 28, no. 1, pp. 127-141, 2009.

[16] N. White, Handbook of Geometric Computing. Springer, 2008, vol. 8, ch. Grassmann-Cayley algebra and robotics applications, pp. 629-656.

[17] D. Kanaan, P. Wenger, S. Caro, and D. Chablat, "Singularity analysis of lower mobility parallel manipulators using Grassmann-Cayley algebra," IEEE Transactions on Robotics, vol. 25, no. 5, pp. 995-1004, 2009.

[18] [Online]. Available: https://hal.archives-ouvertes.fr/hal-01400575 